PROCEEDINGS OF THE

AMERICAN MATHEMATICAL SOCIETY

Volume 138, Number 11, November 2010, Pages 4037-4043

S 0002-9939(2010)10414-8

Article electronically published on May 17, 2010

\title{
EPSILON-HYPERCYCLIC OPERATORS ON A HILBERT SPACE
}

\author{
FRÉDÉRIC BAYART
}

(Communicated by Nigel J. Kalton)

\begin{abstract}
For every fixed $\varepsilon>0$, we construct a bounded linear operator on the separable Hilbert space having an orbit which intersects every cone of aperture $\varepsilon>0$, but such that every orbit avoids a certain ball of positive radius (which depends on the orbit) and a fixed centre.
\end{abstract}

\section{INTRODUCTION}

Let $X$ be a (real or complex) Banach space and let $T \in \mathfrak{L}(X)$ be a bounded operator on $X$. T is called hypercyclic provided there exists a vector $x \in X$ such that its $T$-orbit $O(x, T)$ defined by $O(x, T)=\left\{T^{n} x \mid n \geq 0\right\}$ is dense in $X$. Hypercyclicity is a part of linear dynamics, a rapidly evolving branch of functional analysis. For a complete account on this topic, we refer to the recent book 22.

One theme of linear dynamics is the study of orbits which satisfy a property weaker than denseness. Does this imply that the orbit is itself dense? Does this imply that the operator is hypercyclic? Let us mention here three results:

(1) A somewhere dense orbit must be dense: this is a beautiful result of P. Bourdon and N. Feldman 3 .

(2) Let $d>0$ be such that $O(x, T)$ meets every ball of radius $d$. Then $O(x, T)$ is not necessarily dense; however, $T$ is hypercyclic, so that there exists a dense orbit. This a result of N. Feldman [5].

(3) Even if $T$ admits a weakly dense orbit, $T$ does not need to be hypercyclic; examples are given in [4, [6] and in [2.

In a recent paper [1, C. Badea, S. Grivaux and V. Müller have investigated a weaker version of Feldman's result:

Definition 1.1. Let $\varepsilon \in(0,1)$. A vector $x \in X$ is called an $\varepsilon$-hypercyclic vector for $T \in \mathfrak{L}(X)$ provided that for every nonzero vector $y \in X$, there exists an integer $n \in \mathbb{N}$ such that $\left\|T^{n} x-y\right\| \leq \varepsilon\|y\|$. The operator $T$ is called $\varepsilon$-hypercyclic if it admits an $\varepsilon$-hypercyclic vector.

Thus, an $\varepsilon$-hypercyclic operator admits an orbit which intersects every cone of aperture $\varepsilon$. In [1], the following is proved.

Theorem 1.2. For every $\varepsilon \in(0,1)$, there exists an $\varepsilon$-hypercyclic operator on the space $\ell^{1}(\mathbb{N})$ which is not hypercyclic.

Received by the editors June 16, 2009 and, in revised form, January 20, 2010.

2010 Mathematics Subject Classification. Primary 47A16, 47 B37.

Key words and phrases. Hypercyclic operators, operator weighted shifts.

(C)2010 American Mathematical Society 
It is rather natural that it is easier to produce such an operator on $\ell^{1}$. Indeed this space often plays an extremal role in linear dynamics (see for instance Chapters 2, 4 and 12 of [2]). In [1, the authors asked for the existence of an $\varepsilon$-hypercyclic operator, yet not hypercyclic, on the separable Hilbert space. The aim of this paper is to answer this question positively.

Theorem 1.3. For every $\varepsilon \in(0,1)$, there exists an $\varepsilon$-hypercyclic operator on the separable Hilbert space which is not hypercyclic.

Of course, the existence of such an operator on every separable Banach space remains open. The rest of this paper will be devoted to the proof of Theorem 1.3. It follows the lines of [1, with several modifications due to the Hilbertian nature of the example. However, because of the technical difficulties of these proofs, we provide a completely self-contained argument. See Remark 5.1 for an overview of the differences with [1].

Let us conclude this introduction by showing that Theorem 1.3 is in some sense optimal.

Proposition 1.4. Let $F:(0,+\infty) \rightarrow(0,+\infty)$ be such that $F(r) / r \rightarrow 0$ as $r \rightarrow \infty$. Let $T \in \mathfrak{L}(X)$ and $x \in X$ be such that for any nonzero vector $y \in X$, one may find $n \in \mathbb{N}$ such that $\left\|T^{n} x-y\right\| \leq F(\|y\|)$. Then $T$ is hypercyclic.

Proof. Let $\varepsilon \in(0,1), r_{0}>0$. Also let $x \in X$ be as in the statement of the proposition. There exists $M \in(0, \infty)$ such that $F(M r) / M r<\varepsilon$ for any $r \geq r_{0}$. Let $y \in X$ with $\|y\| \geq r_{0}$ and $n \in \mathbb{N}$ be such that $\left\|T^{n} x-M y\right\| \leq F(M\|y\|)$. Then, setting $z:=M^{-1} x$, one obtains

$$
\left\|T^{n} z-y\right\| \leq \frac{1}{M} F(M\|y\|) \leq \varepsilon\|y\| .
$$

Thus, $T$ has the following property: for any $\varepsilon>0$ and any $r_{0}>0$, there exists a vector $z \in X$ such that for any $y \in X$ with $\|y\| \geq r_{0}$, we may find an integer $n \in \mathbb{N}$ such that $\left\|T^{n} z-y\right\| \leq \varepsilon\|y\|$. A look at the proof of Theorem 1.4 of [1] shows that this forces $T$ to be hypercyclic.

\section{Strategy}

We start with the Hilbert space $H$ which is the $\ell^{2}$-direct sum of countably many copies of $\ell^{2}(\mathbb{N})$. We denote by $\left(e_{n}\right)_{n \geq 0}$ the canonical basis of $\ell^{2}(\mathbb{N})$. Let $T$ be the "simplest" hypercyclic operator on $H$, namely the backward weighted shift with operator weights

$$
T\left(x_{0}, x_{1}, \ldots\right)=\left(T_{1} x_{1}, T_{2} x_{2}, \ldots\right),
$$

where $T_{i}=2 I$. If $\left(y^{(k)}\right)$ is a dense sequence of $H$ of finitely-supported vectors (with support say in $\{0, \ldots, k-1\})$, it is well-known to specialists that the sum

$$
x=\sum_{k}\left(T^{-1}\right)^{n_{k}}\left(y^{(k)}\right)
$$

defines a hypercyclic vector for $T$, provided $\left(n_{k}\right)$ is sufficiently fast increasing. Here, $T^{-1}\left(x_{0}, x_{1}, \ldots\right)$ means $\left(0, T_{1}^{-1} x_{0}, T_{2}^{-1} x_{1}, \ldots\right)$.

We will modify slightly the operators $T_{i}$ to be sure that $T$ is not hypercyclic, but remains very close to a hypercyclic operator. For instance, we can ask that $T_{j}\left(e_{0}\right)=e_{0}$ and $T_{j}\left(e_{n}\right)=2 e_{n}$ for any $n \geq 1$. Modified in this way, $T$ becomes nonhypercyclic and the series (11) is not convergent. 
We then arrange $T$ so that it remains $\varepsilon$-hypercyclic. We first set

$$
z^{(k)}=y^{(k)}+\left(c_{0}^{(k)} e_{p_{k}}, \ldots, c_{k-1}^{(k)} e_{p_{k}}, 0, \ldots\right),
$$

where $c_{j}^{(k)}$ is controlled by $\left\|y_{j}^{(k)}\right\|$ and $p_{k}$ is a large integer. We will modifiy $T_{j}$ for $j$ near $n_{k}$ and just on the vector $e_{p_{k}}$ so that $\left(T^{-1}\right)^{n_{k}}\left(z^{(k)}\right)$ becomes small. Thus the series $x=\sum_{k}\left(T^{-1}\right)^{n_{k}}\left(z^{(k)}\right)$ will be convergent and will give an $\varepsilon$-hypercyclic vector.

\section{Outhine}

Let $a$ be a positive integer so that $2^{-a}<\varepsilon$. Let $X$ be the Hilbert space $\ell^{2}(\mathbb{N})$ endowed with the canonical basis $\left(e_{n}\right)_{n \geq 0}$. Let $H$ be the $\ell^{2}$-direct sum of countably many copies of $X$. Let $\left(y^{(k)}\right)_{k \geq 1}$ be a sequence of vectors of $H$ satisfying the following properties:

(i) the set $\left\{y^{(k)} \mid k \geq 1\right\}$ is dense in $H$;

(ii) each $y^{(k)}$ can be written as a sequence $y^{(k)}=\left(y_{0}^{(k)}, \ldots, y_{k-1}^{(k)}, 0, \ldots\right)$, where each $y_{j}^{(k)}$ is a vector of $\ell^{2}$ which is in the linear span of the vectors $e_{i}, 0 \leq i \leq$ $k-1$;

(iii) $\left\|y^{(k)}\right\| \leq 2^{k}$.

We shall construct by induction on $k \geq 1$ two increasing sequences of integers $\left(n_{k}\right)$ and $\left(n_{k}^{\prime}\right)$ such that $n_{k-1}^{\prime}<n_{k} \leq n_{k}^{\prime}$ for every $k \geq 1$, a sequence $\left(S_{j}\right)_{j \geq 1}$ of bounded operators on $X$ (at step $k$, we will produce the operators $S_{n_{k-1}^{\prime}+1}, \ldots, S_{n_{k}^{\prime}}$ ) and a sequence of vectors $\left(z^{(k)}\right) \in H, z^{(k)}=\left(z_{0}^{(k)}, \ldots, z_{k-1}^{(k)}, 0, \ldots\right)$ satisfying the following properties:

(P1): For any $k \geq 1,\left\|z^{(k)}-y^{(k)}\right\| \leq 2^{-a}\left\|y^{(k)}\right\|$.

(P2): Each operator $S_{j}$ is bounded, invertible, upper triangular with $\left\|S_{j}^{-1}\right\| \leq$ 2 .

(P3): $\left\|S_{j} S_{j-1} \ldots S_{1}\right\| \leq M(a)$ for every $j \in \mathbb{N}$, where $M(a)$ is a constant which just depends on $a$.

(P4): $S_{j} e_{0}=e_{0}$ for every $j \geq 1$.

(P5): $\left\|S_{n_{k}+j-p} \ldots S_{j+1} z_{j}^{(k)}\right\| \leq 2^{-k}$ for every $k \geq 1$, every $j=0, \ldots, k-1$ and every $p \leq n_{k-1}$.

(Q1): $S_{n_{k}^{\prime}} \ldots S_{2} S_{1}=I$ for every $k \in \mathbb{N}$.

(Q2): Let $k \geq 1, p>k^{2}$ and $i$ belong to $\left\{n_{k-1}^{\prime}, \ldots, n_{k}^{\prime}-1\right\}$. Then $S_{1}^{-1} \ldots$ $S_{i}^{-1} e_{p}=2^{i-n_{k-1}^{\prime}} e_{p}$

Once this has been done, we define $T$ on $H$ by setting

$$
T\left(x_{0}, x_{1}, \ldots\right)=\left(S_{1}^{-1} x_{1}, S_{2}^{-1} x_{2}, \ldots\right) .
$$

By (P2), $T$ is well-defined, bounded, with $\|T\| \leq 2$. We then set

$$
x^{(k)}=(\underbrace{0, \ldots, 0}_{n_{k}}, S_{n_{k}} \ldots S_{1} z_{0}^{(k)}, S_{n_{k}+1} \ldots S_{2} z_{1}^{(k)}, \ldots, S_{n_{k}+k-1} \ldots S_{k} z_{k-1}^{(k)}, 0, \ldots) .
$$

By (P5) applied with $p=0,\left\|x^{(k)}\right\| \leq k 2^{-k}$, so that one may define the vector

$$
x=\sum_{k=1}^{+\infty} x^{(k)} \text {. }
$$


We claim that $x$ is an $\varepsilon$-hypercyclic vector for $T$. Indeed, let us fix $k \geq 1$. Then

$T^{n_{k}} x^{(j)}=\left\{\begin{array}{cc}0 & \text { if } j<k \\ z^{(k)} & \text { if } j=k \\ \underbrace{0, \ldots, 0}_{n_{j}-n_{k}}, S_{n_{j}-n_{k}} \ldots S_{1} z_{0}^{(k)}, \ldots, S_{n_{j}-n_{k}+k-1} \ldots S_{k} z_{k-1}^{(k)}, 0, \ldots) & \text { otherwise. }\end{array}\right.$

By (P1) and (P5), this yields

$$
\begin{aligned}
\left\|T^{n_{k}} x-y^{(k)}\right\| & \leq\left\|z^{(k)}-y^{(k)}\right\|+\sum_{j>k} j 2^{-j} \\
& \leq 2^{-a}\left\|y^{(k)}\right\|+\varepsilon_{k},
\end{aligned}
$$

with $\varepsilon_{k} \rightarrow 0$ as $k \rightarrow+\infty$. Let us now fix $y \in H \backslash\{0\}$, and let us consider a subsequence $\left(y^{\left(p_{k}\right)}\right)$, with $p_{k} \rightarrow+\infty$, such that $y^{\left(p_{k}\right)} \rightarrow y$. We get, provided $k$ is large enough,

$$
\begin{aligned}
\left\|T^{n_{p_{k}}} x-y\right\| & \leq\left\|T^{n_{p_{k}}} x-y^{\left(p_{k}\right)}\right\|+\left\|y^{\left(p_{k}\right)}-y\right\| \\
& \leq 2^{-a}\left\|y^{\left(p_{k}\right)}\right\|+\varepsilon_{p_{k}}+\left\|y^{\left(p_{k}\right)}-y\right\| \\
& \leq 2^{-a}\|y\|+2^{-a}\left\|y^{\left(p_{k}\right)}-y\right\|+\varepsilon_{p_{k}}+\left\|y^{\left(p_{k}\right)}-y\right\| \\
& \leq \varepsilon\|y\|
\end{aligned}
$$

since $2^{-a}<\varepsilon$. Thus, $x$ is an $\varepsilon$-hypercyclic vector for $T$. On the other hand, $T$ is not hypercyclic. More precisely, for $w \neq\left(e_{0}, 0, \ldots\right),\left(e_{0}, 0, \ldots\right)$ does not belong to the closure of the orbit of $w$. Otherwise, suppose that we can find an increasing sequence $\left(m_{k}\right)$ such that $T^{m_{k}} w$ goes to $\left(e_{0}, 0, \ldots\right)$. Taking the first coordinate, we get that $S_{1}^{-1} \ldots S_{m_{k}}^{-1} w_{m_{k}}$ tend to $e_{0}$. Now, if we write by (P3) and (P4),

$$
\begin{aligned}
\left\|w_{m_{k}}-e_{0}\right\| & =\left\|S_{m_{k}} \ldots S_{1}\left(S_{1}^{-1} \ldots S_{m_{k}}^{-1} w_{m_{k}}-e_{0}\right)\right\| \\
& \leq M(a)\left\|S_{1}^{-1} \ldots S_{m_{k}}^{-1} w_{m_{k}}-e_{0}\right\|,
\end{aligned}
$$

then we find that $\left(w_{m_{k}}\right)$ goes to $e_{0}$. This contradicts that $w \in H$.

Observe that during the proof that $T$ is $\varepsilon$-hypercyclic yet not hypercyclic, we did not use properties (Q1) and (Q2). They are just useful inside the inductive process. Observe also that the deduction of Theorem 1.3 from properties $(\mathbf{P x})$ is completely similar to the process followed in [1].

\section{The COnstruction}

So, let us start with the construction by setting $n_{0}=n_{0}^{\prime}=0$. Let $k \geq 1$ and let us assume that the construction has been carried out until step $k-1$. Namely, we suppose that we have constructed $z^{(1)}, \ldots, z^{(k-1)}, n_{0}, \ldots, n_{k-1}, n_{0}^{\prime}, \ldots, n_{k-1}^{\prime}$, $S_{1}, \ldots, S_{n_{k-1}^{\prime}}$ so that all the properties (P1) to (P6), (Q1) and (Q2) are satisfied for the integers for which they are meaningful. Let $\Delta_{k}$ be a very large integer. We set

$$
\left\{\begin{array}{l}
n_{k}=n_{k-1}^{\prime}+(a+1)+\Delta_{k} \\
n_{k}^{\prime}=n_{k}+(a+1)+\Delta_{k} .
\end{array}\right.
$$

Let us fix $j \leq k-1$ and let us set $u_{j}^{(k)}=S_{1}^{-1} \ldots S_{j}^{-1} y_{j}^{(k)}$. This is indeed possible since $k-1 \leq n_{k-1}^{\prime}$ and each $S_{l}$ has been supposed to be invertible for $l \leq n_{k-1}^{\prime}$. Let us also consider $l_{j}$ to be the unique integer such that $n_{l_{j}}^{\prime} \leq j<n_{l_{j}+1}^{\prime}$. As 
before, $l_{j}<k$. Writing by (Q1) $u_{j}^{(k)}=S_{n_{l_{j}}^{\prime}+1}^{-1} \ldots S_{j}^{-1} y_{j}^{(k)}$ and using (P2), we get $\left\|u_{j}^{(k)}\right\| \leq 2^{j-n_{l_{j}}^{\prime}}\left\|y_{j}^{(k)}\right\|$. We now decompose $u_{j}^{(k)}$ as

$$
u_{j}^{(k)}:=c_{j}^{(k)} e_{0}+w_{j}^{(k)} \text {, with } w_{j}^{(k)} \in \operatorname{span}\left(e_{1}, \ldots, e_{k-1}\right)
$$

(recall that the operators $S_{j}$, hence $S_{j}^{-1}$, are upper-triangular). This leads us to the following definition:

$$
z_{j}^{(k)}:=y_{j}^{(k)}+2^{-\left(j-n_{l_{j}}^{\prime}\right)} 2^{-a} c_{j}^{(k)} e_{k^{2}} .
$$

In particular, $\left\|z_{j}^{(k)}-y_{j}^{(k)}\right\| \leq 2^{-a}\left\|y_{j}^{(k)}\right\|$, which shows that (P1) is satisfied by the very definition of $z^{(k)}:=\left(z_{0}^{k}, \ldots, z_{k-1}^{(k)}, 0, \ldots\right)$.

We now define the operators $S_{j}, n_{k-1}^{\prime}<j \leq n_{k}^{\prime}$, by giving their action on the vectors of the Hilbertian basis $\left(e_{i}\right)_{i \geq 0}$ of $\ell^{2}$.

- For $i=0, S_{j} e_{0}=e_{0}$.

- For $i=k^{2}$,

$$
S_{j} e_{k^{2}}=\left\{\begin{array}{cl}
2 e_{k^{2}} & j=n_{k-1}^{\prime}+1, \ldots, n_{k-1}^{\prime}+a \\
-e_{0}+e_{k^{2}} & j=n_{k-1}^{\prime}+a+1 \\
\frac{1}{2} e_{k^{2}} & j=n_{k-1}^{\prime}+a+2, \ldots, n_{k-1}^{\prime}+(a+1)+\Delta_{k}=n_{k} \\
2 e_{k} & j=n_{k}+1, \ldots, n_{k}+\Delta_{k} \\
e_{0}+e_{k^{2}} & j=n_{k}+\Delta_{k}+1 \\
\frac{1}{2} e_{k} & j=n_{k}+\Delta_{k}+2, \ldots, n_{k}+\Delta_{k}+a+1=n_{k}^{\prime} .
\end{array}\right.
$$

- For $i \neq 0, k^{2}$,

$$
S_{j} e_{i}=\left\{\begin{array}{cc}
\frac{1}{2} e_{i} & j<n_{k}^{\prime} \\
2^{n_{k}^{\prime}-n_{k-1}^{\prime}-1} e_{i} & j=n_{k}^{\prime}
\end{array}\right.
$$

The successive values of $S_{j} \ldots S_{1} e_{k^{2}}$ for $j=n_{k-1}^{\prime}, \ldots, n_{k}^{\prime}$ are very important. Using (Q1) again to replace $S_{j} \ldots S_{1} e_{k^{2}}$ by $S_{j} \ldots S_{n_{k-1}^{\prime}+1} e_{k^{2}}$, we find that they are equal to

$$
\begin{array}{cccc}
e_{k^{2}}, \ldots, 2^{a} e_{k^{2}}, & 2^{a} e_{k^{2}}-2^{a} e_{0}, \ldots, & 2^{-\Delta_{k}+a} e_{k^{2}}-2^{a} e_{0}, \ldots, & 2^{a} e_{k^{2}}-q 2^{a} e_{0}, 2^{a} e_{k^{2}}, \ldots, e_{k^{2}} \\
j=n_{k}-\Delta_{k} & j=n_{k} & \uparrow
\end{array}
$$

It remains to prove that all the properties are satisfied with this construction. Before going into any detail, let us notice that, since (Q1) is true at step $k-1$, the formal properties (P4), (Q1) and (Q2) are verified.

\section{Boundedness of $S_{j}$ And $S_{j}^{-1}$ : Proof of (P2) And (P3)}

Each $S_{j}$ is the sum of a bounded diagonal operator and of a finite-rank and strictly upper triangular operator. Then, it is bounded and invertible. Moreover, for $j \neq n_{k-1}^{\prime}+a+1$ and $j \neq n_{k}+\Delta_{k}+1$, it is even diagonal, with diagonal terms greater than $1 / 2$. This yields (P2) except for the previous critical indices. However, for these indices, it is not hard to compute $S_{j}^{-1}$ :

$$
S_{j}^{-1}\left(e_{i}\right)=\left\{\begin{array}{cl}
e_{0} & i=0 \\
e_{k^{2}} \pm e_{0} & i=k^{2} \\
2 e_{i} & \text { otherwise }
\end{array}\right.
$$


It is now straightforward to check that $\left\|S_{j}^{-1}\right\| \leq 2$ :

$$
\begin{aligned}
\left\|S_{j}^{-1}\left(\sum_{i} c_{i} e_{i}\right)\right\| & =\left|c_{0}+c_{k^{2}}\right|^{2}+\left|c_{k^{2}}\right|^{2}+4 \sum_{i \neq 0, k^{2}}\left|c_{i}\right|^{2} \\
& \leq 2\left|c_{0}\right|^{2}+3\left|c_{k^{2}}\right|+4 \sum_{i \neq 0, k^{2}}\left|c_{i}\right|^{2} \\
& \leq 4\left\|\sum_{i} c_{i} e_{i}\right\|^{2} .
\end{aligned}
$$

Remark 5.1. Here is the key point where our proof differs from that of [1. It is easier to prove that an operator is bounded on $\ell^{1}$ than on $\ell^{2}$. We had to change $S_{j}$ to ensure that $S_{j}^{-1}$ admits at most two upper-diagonal coefficients. This implies several modifications elsewhere, as in the definition of $z_{j}$, to keep properties $(\mathbf{P} \mathbf{x})$ and $(\mathbf{Q x})$.

To prove (P3) for $j=n_{k-1}^{\prime}, \ldots, n_{k}^{\prime}$, we have to take into account the values of $S_{j} \ldots S_{1}\left(e_{k^{2}}\right)$. These values have been computed above and we find

$$
S_{j} \ldots S_{1}\left(e_{i}\right)=\left\{\begin{array}{cl}
e_{0} & i=0 \\
\alpha e_{k^{2}}+\beta e_{0} & i=k^{2} \\
\gamma e_{i} & \text { otherwise }
\end{array}\right.
$$

with $|\alpha|,|\beta| \leq 2^{a}$ and $|\gamma| \leq 1$. Arguing as before, we easily get (P3).

\section{ACtion of $S_{j}$ ON $z^{(k)}$ : ProOF of (P5)}

We now verify that (P5) holds true. We first write

$$
\begin{aligned}
& S_{n_{k}+j-p} \ldots S_{j+1}\left(z_{j}^{(k)}\right) \\
& \quad=S_{n_{k}+j-p} \ldots S_{1}\left(S_{1}^{-1} \ldots S_{j}^{-1}\right)\left(z_{j}^{(k)}\right) \\
& \quad=S_{n_{k}+j-p} \ldots S_{n_{k-1}^{\prime}+1}\left(S_{1}^{-1} \ldots S_{j}^{-1}\right)\left(y_{j}^{(k)}+c_{j}^{(k)} 2^{-\left(j-n_{l_{j}}^{\prime}\right)} 2^{-a} e_{k^{2}}\right) \\
& \quad=S_{n_{k}+j-p} \ldots S_{n_{k-1}^{\prime}+1}\left(c_{j}^{(k)} e_{0}+w_{j}^{(k)}+c_{j}^{(k)} 2^{-a} e_{k^{2}}\right),
\end{aligned}
$$

where the last line comes from (Q2). We now observe that $|j-p| \leq \max (j, p) \leq$ $n_{k-1}^{\prime}$, and we suppose that $\Delta_{k}$ is very large with respect to $n_{k-1}^{\prime}$. This gives

$$
\begin{aligned}
S_{n_{k}+j-p} \ldots S_{j+1}\left(z_{j}^{(k)}\right)= & c_{j}^{(k)} e_{0}+2^{-\left(n_{k}+j-p-n_{k-1}^{\prime}\right)} w_{j}^{(k)} \\
& +c_{j}^{(k)} 2^{-a}\left(2^{-\Delta_{k}+a+|j-p|} e_{k^{2}}-2^{a} e_{0}\right) \\
= & 2^{-\Delta_{k}-(a+1)-(j-p)} w_{j}^{(k)}+c_{j}^{(k)} 2^{-\Delta_{k}+|j-p|} e_{k^{2}} .
\end{aligned}
$$

We can now adjust $\Delta_{k}$ to be large enough so that $\left\|S_{n_{k}+j-p} \ldots S_{j+1}\left(z_{j}^{(k)}\right)\right\| \leq 2^{-k}$. This achieves the proof of Theorem 1.3.

\section{REFERENCES}

[1] C. Badea, S. Grivaux, and V. Müller. Epsilon-hypercyclic operators. Erg. Th. Dyn. Systems, to appear.

[2] F. Bayart and É. Matheron. Dynamics of linear operators, volume 179 of Cambridge Tracts in Math., Cambridge University Press, 2009. MR2533318

[3] P. S. Bourdon and N. S. Feldman. Somewhere dense orbits are everywhere dense. Indiana Univ. Math. J., 52:811-819, 2003. MR.1986898(2004d:47020)

[4] K. C. Chan and R. Sanders. A weakly hypercyclic operator that is not norm hypercyclic. J. Operator Theory, 52:39-59, 2004. MR2091459 (2005e:47017) 
[5] N. S. Feldman. Perturbations of hypercyclic vectors. J. Math. Anal. Appl., 273:67-74, 2002. MR.1933016 (2003h:47012)

[6] S. Shkarin. Non-sequential weak supercyclicity and hypercyclicity. J. Funct. Anal., 242:37-77, 2007. MR.2274015 (2007i:47010)

Laboratoire de Mathématiques, Université Blaise Pascal, Campus des Cézeaux, F-63177 Aubière Cedex, France

E-mail address: Frederic.Bayart@math.univ-bpclermont.fr 